\title{
Articuler vie professionnelle, familiale et privée aux Pays-Bas : quelle flexibilité pour les salariés?
}

Susanne Burri

\section{(2) OpenEdition}

1 Journals

\section{Édition électronique}

URL : https://journals.openedition.org/rdctss/1973

DOI : 10.4000/rdctss. 1973

ISSN : 2262-9815

Éditeur

Centre de droit comparé du travail et de la sécurité sociale

\section{Édition imprimée}

Date de publication : 1 avril 2018

Pagination : $32-45$

ISSN : 2117-4350

\section{Référence électronique}

Susanne Burri, «Articuler vie professionnelle, familiale et privée aux Pays-Bas : quelle flexibilité pour les salariés ? », Revue de droit comparé du travail et de la sécurité sociale [En ligne], 1 | 2018, mis en ligne le 01 novembre 2021, consulté le 12 novembre 2021. URL : http://journals.openedition.org/rdctss/ 1973 ; DOI : https://doi.org/10.4000/rdctss.1973

\section{cc)}

Revue de droit comparé du travail et de la sécurité sociale est mise à disposition selon les termes de la Licence Creative Commons Attribution - Pas d'Utilisation Commerciale - Pas de Modification 4.0 International. 


\section{ARTICULER VIE PROFESSIONNELLE, FAMILIALE ET PRIVÉE AUX PAYS-BAS: QUELLE FLEXIBILITÉ POUR LES SALARIÉS ?*}

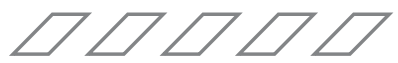

\section{RÉSUMÉ}

Les salariés aux Pays-Bas ont le droit de modifier leur durée, temps et lieu de travail sous certaines conditions, entre autres en vue de faciliter l'articulation de leur vie professionnelle et privée. Ces droits sont plus conséquents que ceux proposés par la Commission européenne. Mais, en termes de congés, le droit néerlandais devrait être adapté si la proposition de directive sur le sujet était adoptée.

MOTS CLÉS: Marché du travail, flexibilité au travail, articulation vie professionnelle et vie privée, proposition de directive de l'UE, congés.

\footnotetext{
ABSTRACT

Workers in the Netherlands have the right to adjust their working time, hours and place under certain conditions, in particular in view of facilitating their work- life balance. These rights are stronger than those recently proposed by the European Commission. However, as regards leaves, Dutch law would have to be amended if the proposal for a directive would be adopted.

KEY WORDS: Labour Market, Flexible Working Arrangements, Work-Life Balance, Proposal for a EU Directive, Leaves.
} 


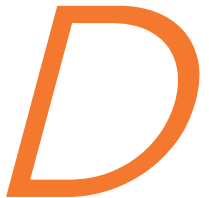

epuis 2000, le droit néerlandais offre des possibilités aux salariés de diminuer ou d'augmenter leur durée du travail, sauf si des intérêts graves de l'entreprise ou du service s'y opposent. Aujourd'hui, les salariés peuvent également demander un aménagement du temps et du lieu de travail. Ces droits ont pour but de permettre aux salariés d'ajuster leur travail à leurs besoins et désirs durant leur carrière, principalement en vue de faciliter l'articulation de la vie professionnelle, familiale et privée. Ce sont ces droits qui seront ici envisagés ainsi que le contentieux y ayant trait.

Depuis le début des années 90, le vieillissement de la population influence négativement la participation au marché du travail néerlandais comme dans certains autres pays de L'UE. Augmenter cette participation constitue un des principaux buts des politiques sociales des Pays-Bas et elle a effectivement augmenté cette dernière décennie. Cette croissance est surtout due aux femmes: leur participation au marché du travail nette est passée de 64,4\% en 2006 à 70,1 \% en 2016. Celle des hommes n'a, par contre, presque pas augmenté (de 79,0\% en 2006 à 79,6\% en 2016) 1 . L'évolution du marché du travail néerlandais est, de plus, caractérisée par une forte amplification de la flexibilité qui prend principalement deux formes: un nombre croissant de salariés travaillant sous contrat à durée déterminée et d'entrepreneurs sans personnel².

Le travail à temps partiel est très répandu aux Pays-Bas. En 2015, 73 \% des femmes et $21 \%$ des hommes étaient à temps partiel. Les femmes travaillent le plus souvent entre 20 et 27 heures par semaine ou à temps plein ( 35 heures par semaine ou plus). Moins d'un quart des femmes travaillent 20 heures par semaine au maximum. Quant aux hommes, $79 \%$ sont à temps plein et environ $10 \%$ entre 28 et 34 heures par semaine. Seulement $7 \%$ des hommes travaillent moins de 20 heures par semaine.

Plus d'un tiers des femmes réduisent leur durée du travail après la naissance d'un enfant, ce qui est très peu le cas des hommes. Beaucoup d'hommes réduisent leur durée du travail en fin de carrière, après $60 \mathrm{ans}^{3}$. Les mères avec enfants et un conjoint dans le même ménage avaient en 2015 un travail rémunéré entre 24 et 26 heures par

1 B. van Hulst, M. Olsthoorn, A. Roeters, "Betaald werk en zorgtaken", in R. Bijl, J. Boelhouwer, A. Wennekers (red.), De sociale staat van Nederland 2017, La Haye, SCP, décembre 2017, p. 148, https://www.scp.nl/Publicaties/Alle publicaties/Publicaties 2017/De sociale staat van Nederland 2017 Depuis le début des années 90, la participation au marché du travail des personnes âgées a également augmenté considérablement.

2 B. van Hulst, M. Olsthoorn \& A. Roeters, "Betaald werk en zorgtaken", in R. Bijl, J. Boelhouwer \& A. Wennekers (red.), De sociale staat van Nederland 2017, La Haye, SCP, décembre 2017, p. 140, https://www.scp.nl/Publicaties/Alle publicaties/Publicaties 2017/De sociale staat van Nederland 2017

3 H.-J. Dirven, W. Portegijs, M. Cuijpers, "Arbeid", in W. Portegijs \& M. van den Brakel (dir.), Emancipatiemonitor 2016, La Haye, SCP, CBS, décembre 2016, p. 66-67. 
semaine, les pères dans la même situation travaillaient environ 40 heures par semaine 4 . Les mères en particulier aimeraient travailler davantage d'heures à mesure que les enfants grandissent ${ }^{5}$.

Une esquisse des politiques des Pays-Bas et de l'UE en vue de faciliter l'articulation de la vie professionnelle et privée permet de placer la législation sur l'aménagement de la durée, le temps et le lieu de travail dans un contexte juridique plus vaste (I). Après une brève ébauche du droit à l'aménagement de la durée du travail, une analyse de la récente loi sur le travail flexible sera présentée (II). Les modalités de cette loi semblent bien correspondre au contexte néerlandais et peuvent - du moins potentiellement faciliter l'articulation de la vie professionnelle, familiale et privée.

\section{I - POLITIQUES EN VUE DE FACILITER L'ARTICULATION VIE PROFESSIONNELLE/VIE PRIVÉE}

\section{A - LE CONTEXTE NÉERLANDAIS}

Depuis le début des années 90, une politique a été menée pour faciliter l'articulation entre la vie professionnelle et la vie familiale et privée, en particulier pour stimuler la participation sur le marché du travail des femmes. Les partenaires sociaux néerlandais ont joué un rôle primordial dans ce développement, au centre duquel se trouvait l'enjeu de la flexibilité. Comment marier les intérêts des salariés d'une part, qui souhaitent adapter leur temps de travail à leur vie personnelle, sans que cela porte atteinte à la sécurité de leur emploi et à leurs conditions de travail? D'autre part, les employeurs sont confrontés à des problèmes de continuité de la production, de prévisibilité des horaires et de flexibilisation de la main-d'œuvre.

Plusieurs lois touchent à ces sujets, par exemple sur le temps du travail, et certaines ontété adoptées spécifiquement en vue de faciliter l'articulation de la vie professionnelle et privée. Dans le cadre de la présente contribution, la loi sur l'adaptation de la durée du travail (Wet aanpassing arbeidsduur, WAA) qui entra en vigueur en 2000 est la plus importante. Cette loi a été plusieurs fois amendée et est maintenant devenue la loi sur le travail flexible (Wet flexibel werken, WFW).

Mais pour qui veut concilier vie professionnelle et vie familiale, certains congés (par exemple le congé maternité, paternité, parental ou pour soins) sont également importants. En 2001, la loi sur l'articulation de la vie professionnelle et la vie privée fût adoptée (Wet arbeid en zorg, WAZO) et révisée plusieurs fois depuis ${ }^{6}$. De plus, la loi sur le temps de travail (Arbeidstijdenwet, ATW) oblige l'employeur à tenir compte des

4 H.-J. Dirven, W. Portegijs, M. Cuijpers, "Arbeid", in W. Portegijs, M. van den Brakel (dir.), Emancipatiemonitor 2016, La Haye, SCP, CBS, décembre 2016, p. 70.

5 H.-J. Dirven, W. Portegijs, M. Cuijpers, "Arbeid", in W. Portegijs, M. van den Brakel (dir.), Emancipatiemonitor 2016, La Haye, SCP, CBS, décembre 2016, p. 72.

6 Voir par exemple S. Burri, A.Ph.C.M. Jaspers, "La durée du travail aux Pays-Bas: Marier la flexibilité de la durée du travail et la sécurité, une gageure impossible? », in I. Daugareilh, P. Iriart (Dir.), Leçons d'une réduction de la durée du travail, pp. 187-20, Pessac, Maison des Sciences de l'Homme d'Aquitaine, 2004. 
circonstances personnelles des salariés lorsqu'il détermine le temps de travail, pour autant qu'on puisse raisonnablement l'exiger de lui ${ }^{7}$. Ce dernier critère semble donc plutôt tenir compte des intérêts de l'employeur, ce qui peut atténuer considérablement l'impact réel d'une telle disposition. L'obligation de tenir compte des responsabilités du salarié en dehors du travail s'applique également aux horaires individuels ${ }^{8}$. De plus, le salarié peut demander un changement de son horaire de travail durant une période d'une année après avoir pris un congé parental ${ }^{9}$.

\section{B - L'AGENDA DE L'UE}

Ces deux sujets - congés et flexibilité - en vue d'une meilleure articulation entre vie professionnelle et vie privée sont, actuellement, fermement ancrés dans les politiques de I'UE dans le cadre du socle européen des droits sociaux ${ }^{10}$. Un des vingt principes clés de ce socle concerne l'équilibre entre la vie professionnelle et la vie privée (principe 9) ${ }^{11}$. Ce principe est rédigé comme suit:

Les parents et les personnes ayant des responsabilités familiales ont droit à des congés appropriés, à des régimes de travail flexibles et à un accès à des services de garde d'enfants. Les femmes et les hommes doivent bénéficier d'un accès égal aux congés spéciaux afin de s'acquitter de leurs responsabilités familiales et sont encouragés à les utiliser de manière équilibrée.

Ce principe n'établit pas de droits spécifiques. Mais la Commission européenne a publié en avril 2017 une proposition de directive concernant l'équilibre entre vie professionnelle et vie privée des parents et aidants ${ }^{12}$. Une disposition est inclue dans cette proposition concernant une «formule souple de travail». Celle-ci offrirait la possibilité pour les travailleurs d'aménager leurs rythmes de travail, y compris par le recours au travail à distance, à des horaires de travail souples ou à une réduction du temps de travail (article 3(f)). L'article 9 préconise ces formules souples de travail:

1. Les États membres prennent les mesures nécessaires pour que les travailleurs ayant des enfants jusqu'à un âge déterminé, qui ne peut être inférieur à douze ans, et les aidants aient le droit de demander des formules souples de travail dans le but de s'occuper de leurs proches. La durée de ces formules souples de travail peut faire l'objet d'une limitation raisonnable.

7 Article 4: 1 ATW. La législation est publiée sur: www.wetten.nl (en néerlandais).

8 Ces responsabilités sont en partie définies, il s'agit en tous cas de responsabilités pour enfants, membres de famille, de parents ou proches dépendants et responsabilités sociales: article 4 : 1 a ATW.

9 Article $4: 1 \mathrm{~b}$ ATW.

10 Voir par exemple, E. Caracciolo di Torella, «An emerging right to care in the EU: a "New Start to Support Work-Life Balance for Parents and Carers", ERA Forum, 2017, n 18, p. 187-198.

11 https://ec.europa.eu/commission/priorities/deeper-and-fairer-economic-and-monetaryunion/european-pillar-social-rights/european-pillar-social-rights-20-principles $\mathrm{fr}$,

12 COM (2017) 253 Proposition de Directive du Parlement européen et du Conseil concernant l'équilibre entre vie professionnelle et vie privée des parents et aidants et abrogeant la directive 2010/18/UE du Conseil. 
2. L'employeur examine les demandes de formules souples de travail visées au paragraphe 1 et y répond, en tenant compte de ses propres besoins et de ceux des travailleurs. L'employeur justifie tout refus d'une telle demande.

3. Lorsque les formules souples de travail visées au paragraphe 1 sont limitées dans la durée, le travailleur a le droit de revenir à son rythme de travail de départ à la fin de la période convenue. Le travailleur a aussi le droit de demander à revenir à son rythme de travail de départ dès lors qu'un changement de circonstances le justifie. L'employeur est tenu d'examiner ces demandes et d'y répondre, en tenant compte de ses propres besoins et de ceux des travailleurs.

Actuellement, le Conseil de l'UE négocie cette proposition de directive. Les avis du Comité des régions et du Comité économique et social européens ont été publiés ${ }^{13}$. II n'est bien sûr pas certain que cette proposition soit adoptée. Elle introduirait en droit de I'UE dans sa forme actuelle deux nouveaux congés (un congé de paternité de dix jours par naissance et de cinq jours par an pour soins à donner, donc un congé d'aidant). Les travailleurs auraient droit à un revenu adéquat pour trois congés encore non payés en droit de l'UE: le congé de paternité, le congé parental et le congé d'aidant. L'adoption de cette proposition de directive comporterait des droits bien plus conséquents pour les travailleurs, comparés à ceux existants au niveau de l'UE ${ }^{14}$.

Pour le droit néerlandais, si cette proposition était adoptée, elle aurait des conséquences sur le congé de paternité (actuellement de deux jours payés, suivi de trois jours de congé parental non rémunérés) ${ }^{15}$. De plus, le congé parental non payé correspondant à 26 semaines au plus de la durée du travail du salarié jusqu'à l'âge de huit ans de l'enfant serait rémunéré et pourrait être pris jusqu'à l'âge de douze ans de l'enfant ${ }^{16}$. En ce qui concerne les possibilités d'aménager la durée et le temps de travail, ainsi que le lieu de travail, le droit néerlandais offre plus de possibilités que la proposition de la Commission européenne. Ce thème fait partie déjà depuis des décennies des politiques sociales des Pays-Bas.

13 Voir pour tous les documents disponibles

http://eur-lex.europa.eu/procedure/FR/2017 85?rid=1\&qid=1516354156164, consultée le 19 janvier 2018.

14 Voir par exemple S. Burri, "La conciliation de la vie professionnelle, privée et familiale. L'approche juridique de I'Union européenne», in F. Milewski, H. Périvier (Dir.), Les discriminations entre les femmes et les hommes pp. 253-280, Paris, Presses de Sciences Po, 2011 et European network of legal experts in gender equality and non-discrimination, A. Mc Colgan, Measures to address the challenges of work-life balance in the EU Member States, Iceland, Liechtenstein Norway, Brussels, European Commission, 2015, http://www.equalitylaw. eu/publications/thematic-reports

15 Articles 4:2 et 6:5 (4) Wet arbeid en zorg. Le gouvernement actuel a annoncé que le congé de paternité serait prolongé, mais aucun projet de loi n'a encore été publié. Voir les propositions gouvernementales sur https://www.rijksoverheid.nl/regering/regeringsverklaring

16 Voir pour des rapports sur la transposition des directives européennes au niveau national les publications du réseau d'experts juridiques dans le domaine de l'égalité des genres et de la non-discrimination sur

http://www.equalitylaw.eu/ et http://ec.europa.eu/justice/gender-equality/tools/legal-experts/index en.htm 


\section{II - AMÉNAGEMENT DE LA DURÉE, DU TEMPS ET DU LIEU DE TRAVAIL EN DROIT NÉERLANDAIS}

\section{A - LE RÔLE DES PARTENAIRES SOCIAUX}

La différentiation de la durée du travail fait partie de l'agenda social au niveau national des partenaires sociaux et du gouvernement depuis le début des années 90. En 1993, un accord national a été conclu au sein de la Fondation du Travail (Stichting van de Arbeid), une organisation bipartite. ${ }^{17}$ Cette Fondation recommandait aux partenaires sociaux de réglementer le droit à l'aménagement du temps de travail dans les conventions collectives en vue de favoriser une plus grande différenciation de la durée du travail et du travail à temps partiel. Dans cet accord, la Fondation indique que l'employeur doit accepter une proposition de changement de la durée du travail (que ce soit une diminution ou une augmentation des heures de travail), sauf si un intérêt grave de l'entreprise s'y oppose. La nouvelle loi sur l'aménagement de la durée du travail (WAA) reprendra, sept ans plus tard, précisément cette formulation. Ceci illustre le rôle que les partenaires sociaux peuvent jouer dans la politique sociale. Avant l'accord de 1993, les conventions collectives ne contenaient que peu de clauses relatives à la modification de la durée du travail. Si des clauses concernaient le travail à temps partiel ${ }^{18}$, elles ne mentionnaient qu'en termes généraux la possibilité de travailler à temps partiel. Même après l'accord de 1993, seules quelques conventions reprenaient la formulation de la Fondation du Travail ${ }^{19}$. Vu ce maigre résultat, différents partis politiques ainsi que le gouvernement néerlandais présentèrent des projets de lois. En tout, quatre propositions ont fait l'objet de longs débats politiques; finalement, le projet de loi gouvernemental fût adopté20.

17 Stichting van de Arbeid, Overwegingen en aanbevelingen ter bevordering van deeltijdarbeid en differentiatie in arbeidsduurpatronen, Den Haag, Stichting van de Arbeid, 1993.

18 En vue de renforcer le statut juridique des travailleurs à temps partiel, le législateur néerlandais a adopté en 1996 une loi interdisant de discriminer les salariés et les fonctionnaires en raison de la durée du travail, Stb.1996, 391.

19 En 1998, seulement 14 conventions contenaient une disposition qui s'inspirait de la formulation de l'accord de la Fondation du Travail: Arbeidsinspectie, Deeltijdarbeid in cao's, Den Haag, Ministerie van SZW, 1999.

20 De nombreuses publications ont paru sur cette loi, voir notamment, S. Burri, Tijd delen. Deeltijd, gelijkheid en gender in Europees- en nationaalrechtelijk perspectief, Europese Monografieën 66, Deventer,Kluwer, 2000, p. 589-607; S. Burri, « Pays-Bas: loi sur l'adaptation du temps de travail », Futuribles, Mai 2000, p. 78-79; S. Burri, « Loi sur la conciliation entre la vie privée et la vie professionnelle", Liaisons Sociales Europe, 2001, n.33, p. 6-7; S.W. Kuip, E. Verhulp, Wet aanpassing arbeidsduur, Den Haag, SDU Uitgevers, 2000; D.J.B. de Wolff, Wet aanpassing arbeidsduur, Actualiteiten Sociaal Recht, Deventer, Kluwer, 2000. 


\section{B - LA LOI SUR L'AMÉNAGEMENT DE LA DURÉE DU TRAVAIL}

Cette loi (Wet aanpassing arbeidsduur (WAA)) entra en vigueur en 2000 et avait pour but d'augmenter le nombre de personnes participant au marché du travail, ainsi que de faciliter l'articulation entre travail et vie privée ${ }^{21}$. Elle s'appliquait aux salariés et aux fonctionnaires, qui avaient le droit de demander un aménagement de la durée du travail si leur ancienneté était d'un an au minimum. La demande devait être faite par écrit au moins quatre mois avant la date prévue du changement et devait stipuler la durée et les horaires de travail souhaités. L'employeur ne pouvait refuser le changement proposé que si des intérêts graves de l'entreprise ou du service s'y opposaient. En cas d'acceptation du changement de la durée du travail, l'employeur déterminait la répartition des heures de travail selon les vœux du salarié, sauf si les intérêts de l'entreprise devaient raisonnablement primer sur ceux du salarié. L'intérêt du salarié primait donc en ce qui concerne la durée du travail, mais l'employeur avait plus d'influence sur l'horaire de travail. Le législateur néerlandais a ainsi voulu trouver un équilibre entre les intérêts du salarié et ceux de l'employeur. Une requête ne pouvait être faite qu'une fois tous les deux ans après l'acceptation ou le refus de l'employeur. Cette limitation avait pour objet d'éviter que l'employeur ne soit confronté à de continuels changements de durée du travail. L'évaluation de cette loi en 2003 ne donna lieu à aucun amendement, vu entre autres les conclusions positives de cette évaluation. En pratique, un ajustement de la durée du travail se fait très souvent par accord entre l'employeur et le salarié sans se référer à cette loi. Mais le gouvernement constatait en se basant sur l'évaluation que celle-ci offre le cadre dans lequel une demande doit être accordée ${ }^{22}$.

La loi sur l'aménagement de la durée du travail ne fût partiellement amendée qu'à partir du $1^{\text {er }}$ janvier 2015 en ce qui concerne quelques aspects de procédure visant à faciliter des requêtes de salariés. De plus, un changement de la durée du travail n'a, depuis, plus nécessairement un caractère structurel, car un aménagement de la durée du travail peut également être demandé pour des périodes et une durée du travail variables. ${ }^{23}$ Aujourd'hui, la loi sur le travail flexible offre encore plus de flexibilité aux salariés des secteurs privé et public.

21 Kamerstukken II 1998/99 26358, 3, p. 7; Stb. 2000, 114 \& 115.

22 Kamerstukken II 2003-2004, 29501, 1, p. 1.

23 Wet modernisering verlof en arbeidstijden, Stb. 2014, 565, entrée en vigueur le 1er janvier 2015 et amendée depuis 1er avril 2016, Stb. 2015, 518. Voir S. E. Heeger-Hertter, A.G. Veldman, «'Het nieuwe werken' in nieuwe wetgeving. Flexibilisering van verlof, werktijd en arbeidsplaats", Arbeidsrecht, 2015, p. 28; E.M. Bevers, "de Wet modernisering regelingen voor verlof en arbeidstijden: noodzakelijk en nuttig?", Tijdschrift voor de Arbeidsrechtpraktijk (TAP), 2015, n², p. 60 . 


\section{C - LA LOI SUR LE TRAVAIL FLEXIBLE}

La loi sur le travail flexible (Wet flexibel werken, WFW) a remplacé et partiellement modifié la loi sur l'aménagement de la durée du travail (WAA) décrite ci-dessus ${ }^{24}$. Depuis son entrée en vigueur le $1^{\text {er }}$ janvier 2016, le salarié ou le fonctionnaire a non seulement le droit de demander un aménagement de la durée du travail, mais également du temps de travail ainsi que du lieu de travail. Cette loi est une initiative de deux partis politiques d'opposition: Groen Links (parti de gauche) et CDA (parti chrétien-démocratique du centre). Un des principaux buts de cette loi est de faciliter l'articulation de la vie professionnelle, familiale et privée.

La loi s'applique aux salariés qui ont un contrat de travail (en droit privé ou public) avec un employeur (même si le travail est effectué à l'étranger) ${ }^{25}$. Un intérimaire - qui en droit néerlandais a un contrat de travail avec l'agence intérimaire - ne peut donc pas invoquer cette loi vis-à-vis de l'entreprise ou du service dans lequel il travaille. De même, une personne ayant atteint l'âge de la retraite ne peut invoquer cette loi $^{26}$.

L'article 2 WFW est le plus important. Le premier paragraphe dispose qu'un salarié qui est employé depuis au moins 26 semaines avant la date prévue du changement peut demander un aménagement de la durée du travail, du lieu de travail ou du temps de travail. Cette demande peut concerner diverses périodes et avoir une ampleur différente ou non.

Le salarié a donc maintenant besoin de moins d'ancienneté pour pouvoir faire une demande qu'avant l'entrée en vigueur de la loi sur le travail flexible. En cas de circonstances particulières, une demande peut même être faite avant que la période de 26 semaines ne soit terminée ${ }^{27}$. Le critère décisif étant l'emploi, si le salarié est en congé (par exemple de maternité ou parental), le congé compte pour la période des 26 semaines.

Le libellé concernant les périodes et l'ampleur des changements n'est pas très clair, mais signifie en tous cas que toutes sortes de combinaisons sont possibles. Son importance réside dans le fait que, contrairement, aux dispositions antérieures, un changement temporaire est possible. Par exemple, un salarié travaillant 40 heures par semaine sur cinq jours, peut demander un changement correspondant à 24 heures sur trois jours pour une période d'un an. De tels changements temporaires peuvent également concerner l'horaire (par jour par exemple) ou le lieu de travail. Si un salarié désire des changements fréquents et/ou de courte durée, il pourrait être préférable de demander un ou des congés, car une modification de la durée, du lieu ou du temps de travail implique une modification du contrat de travail, ce qui a des conséquences, par

24 Stb. 2015, 245, 376 \& 464. Voir H.G, van Andel, "Van (wet) aanpassing arbeidsduur naar (wet) flexibel werken", Arbeidsrecht, 2017, n²4; E. Verhulp, "Wet flexibel werken", in J.M. van Slooten, M.S.A. Vegter, E. Verhulp (Dir.), Tekst en commentaar, Arbeidsrecht, Wolters Kluwer, 2016.

25 Kamerstukken II 1998/99 26358, 5, p. 20; article 1 (a) et (b) WFW. Des dispositions spécifiques s'appliquent aux militaires, Stb. 2000, 344 et article 2 (2) WFW.

26 Article 1a WFW. Le but étant d'éviter que des salariés âgés ne puissent demander une augmentation de leur temps de travail et ainsi restreindre les possibilités de travail des jeunes.

27 Kamerstukken II 2014/15, 32889, 20. 
exemple, sur des droits liés aux vacances ou à la pension de retraite ${ }^{28}$. De plus, des changements fréquents risquent de passablement compliquer l'organisation du travail pour l'employeur.

Il est donc possible d'aménager - sous forme de diminution ou d'augmentation - la durée du travail. En cas de diminution de la durée du travail, l'employeur n'a pas l'obligation de remplacer le salarié pour les heures libérées. Si l'employeur ne remplace pas le salarié et que les tâches du salarié ne sont pas adaptées, ceci peut mener à une surcharge de travail.

En ce qui concerne l'augmentation de la durée du travail, elle n'est possible que pour sa propre fonction. Si par exemple, un salarié est enseignant, une augmentation devrait concerner des heures d'enseignement ou des tâches liées à l'enseignement de ce salarié. Il ne sera quelquefois peut-être pas facile de définir clairement les tâches qui correspondent à une fonction déterminée. Dans un tel cas, la description de la fonction dans le contrat de travail sera une indication comme le seront, le cas échéant, les tâches effectuées en pratique par le salarié. Si des heures correspondant à la fonction du salarié sont disponibles, l'employeur devra en tant que «bon employeur ${ }^{29}$ offrir ces heures au salarié, éventuellement en appliquant une procédure de candidature interne ${ }^{30}$.

La demande de modification de la durée, du temps ou du lieu de travail doit se faire par écrit au moins deux mois avant la date prévue du changement, sauf circonstances particulières ${ }^{31}$. Ce délai est donc plus court qu'avant l'entrée en vigueur de la loi sur le travail flexible. La demande doit indiquer la date désirée d'entrée en vigueur des changements, les modifications souhaitées ainsi que l'horaire hebdomadaire. Le salarié n’a pas besoin de justifier sa demande.

Le salarié peut, sauf circonstances particulières, faire une nouvelle demande une année après l'acceptation ou le refus de la demande précédente. Ce délai est également plus court qu'auparavant. Si le salarié retire sa demande d'aménagement de la durée du travail, par exemple parce que l'horaire proposé par l'employeur ne lui convient pas (conformément à l'article 2 (7)), ce délai ne s'applique pas, car il n'est pas question de refuser ou d'accepter le changement de la durée du travail. Si un salarié fait une demande avant l'écoulement du délai d'un an, en cas de changements de circonstances, l'employeur devra évaluer cette demande en «bon employeur » et ne

28 Voir cette suggestion dans S. E. Heeger-Hertter, A.G. Veldman, "Het nieuwe werken' in nieuwe wetgeving. Flexibilisering van verlof, werktijd en arbeidsplaats ", Arbeidsrecht, 2015, $\mathrm{n}^{\circ} 28$, p. 3.

29 C'est une notion générale (article 7:611 Code Civil) qui peut s'appliquer à de nombreuses situations, entre autres en relation avec le temps de travail, voir par exemple, Ktr Alkmaar 10 février 2017, $A R, 2017-0268$.

30 Handelingen II 1998/99, p. 5891.

31 Article 2 (3) WFW. 
pourra la refuser que si des intérêts graves s'y opposent ${ }^{32}$. Le salarié devra, dans ce cas, établir qu'il est question de changement de circonstances.

Lorsqu'un salarié adresse une demande, la loi précise que l'employeur et le salarié doivent se concerter. Si certaines données font défaut dans la requête (par exemple la date souhaitée d'entrée en vigueur du changement), le salarié aura la possibilité de remplir les conditions de procédure prévues par la loi. Le but de la concertation est de trouver une solution acceptable pour les deux parties ${ }^{33}$. Si cette concertation montre que certains vœux du salarié ne seront pas satisfaits, le salarié peut amender ou retirer sa demande.

Une demande de modification de la durée du travail et du temps de travail (horaires) doit en principe être acceptée par l'employeur pour ce qui est de la date prévue du changement et de l'ampleur du changement. Un refus de l'employeur ne peut être justifié que par des intérêts graves de l'entreprise ou du service ${ }^{34}$. La loi donne quelques exemples. Un refus de l'employeur de diminuer la durée du travail est justifié si cette diminution pose de graves problèmes de remplacement, de sécurité ou de planification. Une augmentation de la durée du travail peut être refusée en cas de graves problèmes financiers, d'organisation ou encore par manque de travail ${ }^{35}$. Sur ces points, les dispositions de la loi sur le travail flexible sont restées identiques à celles de la loi sur l'aménagement de la durée du travail.

Le contentieux sur l'application de la loi sur l'aménagement de la durée du travail est rare, et il a principalement pour objet la possibilité de justification par l'employeur d'un refus. Entre le 1er janvier 2005 et le 1er janvier 2015, seulement 47 jugements ont eu trait à la loi sur l'aménagement de la durée du travail ${ }^{36}$. La plupart des questions juridiques concernaient les justifications présentées par les employeurs pour refuser une demande de diminution de la durée du travail ${ }^{37}$. Après une diminution de la durée du travail durant un congé parental, les juges ne sont pas facilement enclins à considérer qu'un refus de la part de l'employeur de modifier de la durée soit justifié après la fin d'un tel congés8.

32 Kamerstukken I/ 1998/99, 26358, 5, p. 9 et Handelingen I 1999/2000, p. 16-634.

33 Article 2 (4) WFW, Kamerstukken II 1998/99, 26358, 3, p. 16.

34 Article 2 (5) WFW.

35 Article 2 (9) et (10) WFW.

36 Voir E. M. Bevers, "de Wet modernisering regelingen voor verlof en arbeidstijden: noodzakelijk en nuttig?", Tijdschrift voor de Arbeidsrechtpraktijk (TAP), 2015, n², p. 60.

37 Sur 47 jugements, 27 concernaient une demande de diminution de la durée du travail. Dans 24 cas, les justifications présentées par les employeurs furent examinées. Sur ces 24 cas, les juges considérèrent 9 fois que les justifications représentaient des intérêts graves de l'entreprise dans le sens de la loi et 15 fois que tel n'était pas le cas. Depuis l'entrée en vigueur de la loi sur le travail flexible, aucun jugement jusqu'en décembre 2017 ne concerne les justifications pour refus de l'employeur.

38 Voir par exemple Ktr. Nijmegen18 juillet 2001, JAR 2001/192 et Ktr.'s-Hertogenbosch, 7 janvier 2003, JAR 2003, p. 64. 
Seuls quelques jugements concernent une demande d'augmentation de la durée du travail ${ }^{39}$. Si, par exemple, un même travail est exécuté par un salarié titulaire d'un contrat de travail à durée indéterminée et des travailleurs sur appel, l'intérêt du salarié ayant un contrat de travail à durée indéterminée qui désire une augmentation de la durée du travail pourrait primer ${ }^{40}$. L'employeur ne peut pas refuser une augmentation de la durée du travail en invoquant le mauvais fonctionnement d'un salarié. Il en irait autrement s'il existe un dossier établissant des dysfonctionnements avant la demande du salarié ${ }^{41}$.

La rareté du contentieux sur ces sujets n'est pas très étonnante vue la culture juridique néerlandaise. En général, il existe peu de contentieux concernant les conditions de travail aussi longtemps que la relation de travail perdure.

Une demande de modification du temps de travail (horaire de travail par semaine ou sur une autre période) ne peut également être refusée que si des intérêts graves de l'entreprise s'y opposent. Quelques justifications possibles d'un refus sont indiquées par la loi. Il doit s'agir de graves problèmes par exemple de sécurité, financiers, $\mathrm{d}^{\prime}$ organisation ou $\mathrm{d}^{\prime}$ horaires $^{42}$. Sur ce point, la loi sur le travail flexible offre un nouveau droit spécifique aux salariés. Avant l'entrée en vigueur de cette loi, il n'existait que l'obligation générale de l'employeur de tenir compte des circonstances personnelles dans le temps de travail (voir I.A.). Un changement de la répartition des heures de travail ne pouvait être demandé qu'en relation à une demande de modification de la durée du travail (voir II.B) ${ }^{43}$.

Une demande de changement de lieu de travail peut être refusée par l'employeur plus facilement. L'employeur doit considérer une demande de modification du lieu du travail et, en cas de refus, se concerter avec le salarié ${ }^{44}$.

L'employeur détermine la répartition des heures de travail selon les vœux du salarié. II n'est pas encore très clair comment les concepts de temps du travail (werktijd) et de répartition des heures de travail (spreiding van uren) seront définis. Ceci pourrait être important en pratique, étant donné que l'employeur peut changer la répartition des heures de travail souhaitée, si son intérêt est tel qu'il doit raisonnablement primer sur celui du salarié ${ }^{45}$. L'employeur communique sa décision suite à une demande de

39 Sur 5 jugements concernant une augmentation de la durée du travail, les juges ont décidé trois fois qu'il était question d'intérêt grave de l'entreprise et deux fois que ce n'était pas le cas. Depuis l'entrée en vigueur de la loi sur le travail flexible seulement un jugement a été publié concernant une augmentation de la durée du travail qui fut refusée à juste titre (Ktr

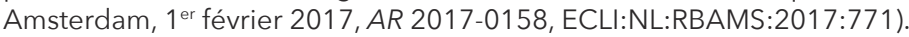

40 Ce fût le cas dans ce jugement: Ktr. Maastricht 2 février 2001, JAR 2001, p. 49.

41 Kamerstukken II 1998/99, 26358, 5, p. 8.

42 Article 2 (11) WFW.

43 Voir par exemple Ktr. Amsterdam 2 mai 2001, JAR 2001/113.

44 Article 2 (6) WFW.

45 Article 2 (7) WFW. 
modification de la durée, du temps ou du lieu de travail par écrit. En cas de refus d'un tel changement (même partiel) ou de la répartition des heures de travail souhaitée, les raisons du refus doivent être également exposées par écrit ${ }^{46}$.

Si l'employeur n'a pas pris de décision un mois avant la date souhaitée de l'entrée en vigueur de la modification ou ne l'a pas communiqué par écrit au salarié, la durée, le temps ou lieu de travail seront alors conformes aux vœux du salarié ${ }^{47}$.

La loi sur le travail flexible offre à l'employeur la possibilité de revenir sur sa décision, une possibilité qui n'existait pas avant l'entrée en vigueur de cette loi48. L'employeur a cette possibilité si et seulement si après sa décision de nouveaux faits et circonstances forment des intérêts graves de l'entreprise ou du service. En outre, l'employeur doit s'entretenir avec le salarié et donner sa décision par écrit en précisant les motifs.

Dans l'intérêt des employeurs, la loi aménage encore d'autres limites. Ainsi, les partenaires sociaux ont la possibilité, par accord collectif, de réglementer différemment, voire d'exclure le droit à l'augmentation de la durée du travail, du changement du temps ou du lieu de travail. Mais aucune dérogation portant atteinte aux droits des salariés en ce qui concerne la diminution de la durée du travail n'est admise. Les conventions collectives peuvent par contre comprendre des clauses plus favorables aux salariés que la loi.

Si aucune convention collective n'est applicable ou si elle ne contient pas de dispositions y relatives, un accord par écrit entre l'employeur et le comité d'entreprise, ou à défaut les représentants du personnel, permet de déroger aux dispositions légales sur le droit d'augmenter la durée du travail, le temps de travail ou de modifier le lieu de travail. Un tel accord est valable cinq ans au plus ${ }^{49}$.

En outre, les entreprises de moins de dix salariés sont exclues du champ d'application de la loi. Elles sont seulement tenues d'introduire une clause sur ce thème dans le règlement intérieur ${ }^{50}$. La loi ne spécifie rien quant au contenu d'une telle clause.

46 Article 2 (8) WFW.

47 Article 2 (12) WFW. Voir par exemple Rb. Oost-Brabant, 27 octobre 2016, ECLI:NL:RBOBR:2016:5951.

48 Kamerstukken II 2014/15, 32889, 25; articles 13 et 14 WFW.

49 Article 2 (15) et (17) WFW. Voir par exemple CRvB 15 septembre 2005, TAR 2006/66.

50 Article 2 (16) WFW. 
L'employeur n'a pas le droit de licencier un salarié parce qu'il a fait une demande de modification de la durée, du temps ou du lieu de travail. Cette interdiction concerne également le non-renouvellement d'un contrat à durée déterminée pour cette raison ${ }^{51}$. Une évaluation de la loi est prévue tous les cinq ans ${ }^{52}$.

\section{Quelques conclusions}

Les salariés jouissent en droit néerlandais de droits conséquents en ce qui concerne l'aménagement de la durée (diminution et augmentation) du travail et du temps de travail. Dans un contexte où le travail à temps partiel domine, c'est souvent par accord entre l'employeur et le salarié qu'un tel ajustement se fait si le salarié le désire. Bien sûr, le contexte culturel de l'organisation et les tâches à remplir jouent également un rôle: il sera plus facile d'adapter durée et temps du travail dans certaines organisations, secteurs ou fonctions que dans d'autres. Mais le peu de contentieux sur l'aménagement de la durée du travail depuis l'entrée en vigueur de la loi (Wet aanpassing arbeidsduur) en 2000 et son évaluation sembleraient indiquer que l'application de cette loi en pratique pose peu de problèmes.

Le développement de la législation sur l'aménagement de la durée, du temps et du lieu de travail reflète une nette tendance vers de plus amples possibilités de flexibilité pour le salarié. Cette tendance se situe dans un contexte dans lequel le travail se fait de plus en plus de manière flexible: "het nieuwe werken » ou «le nouveau travail ». De plus, la proposition de la directive de la Commission européenne reflète une même tendance, en prescrivant en plus des droits aux congés plus conséquents. Sur ce point, les salariés ont, en droit néerlandais, en partie moins de droits que les congés proposés par la Commission européenne. Pour les salariés, prendre des congés surtout lorsqu'ils ont rémunérés - présente de nets avantages car le contrat de travail n'a dans ces cas pas besoin d'être adapté. Par contre, une diminution de la durée du travail aura des conséquences sur la rémunération et d'autres droits (par exemple sur les pensions et certaines allocations). De plus, si le salarié n'est pas remplacé et/ou les tâches adaptées, ceci peut entraîner une surcharge de travail.

Depuis l'entrée en vigueur de la loi sur le travail flexible, le $1^{\text {er }}$ janvier 2016, le droit néerlandais offre aux salariés du secteur privé et public non seulement la possibilité d'ajuster la durée du travail, mais également le temps de travail (en ce qui concerne l'ampleur du changement), sauf si des intérêts graves de l'entreprise ou du service s'y opposent. II n'est donc pas facile pour l'employeur de refuser un tel changement. L'employeur a, par contre, plus d'influence sur la répartition des heures de travail et

51 Kamerstukken II 1998/99, 26358, 5, p. 31. Article 3 WFW. Voir Rb. Midden-Nederland, 11 juin 2003, ECLI:NL:RBMNE:2013: CA2746.

52 Article 4 WFW. La première évaluation de la loi sur l'aménagement de la durée du travail a eu lieu en 2003 (Onderzoek tenbehoeve van de evaluatie van de Waa en de WOA: annexe Kamerstukken I 2003/04, 29503, 1). Selon cette recherche, la plupart des objectifs de cette loi ont été réalisés en pratique. La première évaluation de la loi sur le travail flexible devrait avoir lieu en 2021. 
il faudra voir en pratique comment la nouvelle loi - qui rend également possible un changement de temps du travail - sera interprétée sur ce point. En décembre 2017, aucun contentieux n'avait encore été publié sur cette question.

Le salarié peut maintenant demander une modification de la durée, du temps et du lieu de travail pour des périodes et une ampleur variables. Cette flexibilité accrue permet un changement temporaire, ce qui peut d'une part contribuer à atténuer les risques liés à une réduction définitive des heures de travail. Mais d'autre part, cette possibilité risque de confronter les employeurs à des problèmes d'organisation, surtout si des périodes de changement se succèdent à court terme.

La loi sur le travail flexible est passablement compliquée, du fait que la modification de la durée du travail et du temps du travail peuvent être refusés dans peu de cas, alors qu'en cas d'une demande de changement du lieu de travail et de la répartition des heures de travail l'employeur a plus de possibilités de justifier un refus. Les conventions collectives peuvent contenir des clauses qui peuvent déroger défavorablement aux droits du salarié à l'augmentation de la durée, du changement du temps ou du lieu de travail. De plus, la loi ne s'applique pas aux entreprises de moins de dix salariés, qui doivent introduire une clause sur ce sujet dans leurs règlements intérieurs. Finalement, le droit néerlandais offre des droits conséquents aux salariés qui aimeraient (temporairement ou non) changer la durée, le temps ou le lieu de travail; potentiellement, une telle flexibilité peut réellement contribuer à faciliter l'articulation entre vie professionnelle, familiale et privée.

\section{SUSANNE BURRI}

Professeure associée au département du droit de l'Université d'Utrecht, Pays-Bas.

Thèmes de recherche : Principe d'égalité en droit de I'UE, néerlandais et international, articulation vie professionnelle et privée, relations de travail atypiques, familles et droit.

\section{Publications:}

S. Burri, "Care in Family Relations - The Case of Surrogacy Leave», European Journal of Law Reform, 2015, vol. 17, $n^{\circ} 2$, pp. 271-281.

$\sim$ S. Burri, «Challenging Perspectives on Work/Life Balance Issues in EU and Dutch Law». In T. Addabbo, W. Bromwich, T.M. Fabbri \& I. Senatori (Eds.), «Labour And Social Rights. An Evolving Scenarion, Proceedings of the Twelfth International Conference in Commemoration of Prof Marco Biagi, 2015, Turin, Giappichelli, pp. 155-190. 